\title{
33.
}

\section{Solution générale d'un problème d'analyse combinatoire.}

(Par Mr. Ramus, lecteur en math. de l'université de Copenhague.)

Dans le Bulletin des sciences mathém. etc., févr. 1829., Mr. Cournot a traité le problème suivant, dont celui de Mairan n'est que le cas le plus simple:

„Sur le nombre total des combinaisons qu'on peut faire avec un „, nombre donné de pièces, déterminer séparément le nombre de celles "dont les exposans *), divisés par un certain module $p$, donnent pour reste "l'un des nombres $0,1,2,3, \ldots . p-1$."

La solution de Mr. Cournot est fondée sur la nature des fonctions cherchées $y_{x}^{(0)}, y_{x}^{(1)}, y_{x}^{(2)}, \ldots y_{x}^{(p-1)}$ ( $x$ étant le nombre des pièces $)$ de satisfaire à ces équations linéaires aux différences finies, laccroissement de $x$ étant 1,

$$
\begin{aligned}
& \triangle^{p} y_{x}^{(0)}=y_{x}^{(0)}+1, \\
& \Delta^{p} y_{x}^{(r)}=y_{x}^{(r)},
\end{aligned}
$$

$r$ désignant l'un quelconque des nombres $1,2, \ldots, p-1$. Lintégration complète en est facile, et les constantes arbitraires peuvent être déterminées pour chaque cas particulier. En posant successivement $p=2,=3$, $=4$, il trouve :

1) pour $p=2$, les formules bien connues:

2) pour $p=3$ :

$$
y^{(0)}=2^{x-1}-1, y^{(1)}=2^{x-1} ;
$$

$$
\begin{aligned}
& y^{(0)}=\frac{\pi}{3} 2^{x}+\frac{\pi}{3} \cos \frac{\pi}{3} \pi x-1, \\
& y^{(1)}=\frac{1}{3} 2^{x}-\frac{\pi}{3} \cos \frac{\pi}{3} \pi x+\frac{1}{\sqrt{3}} \sin \frac{\pi}{3} \pi x, \\
& y^{(2)}=\frac{1}{3} 2^{x}-\frac{1}{3} \cos \frac{\pi}{3} \pi x-\frac{1}{\sqrt{3}} \sin \frac{\pi}{3} \pi x ;
\end{aligned}
$$

3) pour $p=4$ :

$$
\begin{aligned}
& y^{(0)}=\frac{1}{4} 2^{x}+\frac{1}{2}(\sqrt{ } 2)^{x} \cos \frac{\pi}{4} \pi x-1, \\
& y^{(1)}=\frac{1}{4} 2^{x}+\frac{1}{2}(\sqrt{ })^{x} \sin \frac{\pi}{4} \pi x, \\
& y^{(2)}=\frac{1}{4} 2^{x}-\frac{1}{2}(\sqrt{ } 2)^{x} \cos \frac{1}{4} \pi x, \\
& y^{(3)}=\frac{\pi}{4} 2^{x}-\frac{1}{2}(\sqrt{ } 2)^{x} \sin \frac{\pi}{4} \pi x .
\end{aligned}
$$

-) Mr. Cournot appelle $n$ l'exposant d'une combinaison $n$ à $n$. 
Enfin il ajoute:, il serait curieux de savoir si, en général, les termes ", affectés de $2^{x}$ ont, dans toutes les fonctions $y_{x}$ des coefficients égaux en,tr'eux et à $\frac{1}{p}$; et si lorsque le nombre $p$ n'est pas premier, il y a tou„jours un certain nombre de termes qui disparaissent de la valeur de $y_{x}$."

Le but de cet article est d'offrir la solution générale du problème qu'on peut trouver sans difficulté en suivant une voie différente.

En effet la solution générale est contenue dans ces deux formules:

$$
\begin{aligned}
& \text { (A.) } y_{x}^{(0)}=\frac{2^{x}}{p}+\frac{2}{p} \Sigma\left(2 \cos \frac{k \pi}{p}\right)^{x} \cos \frac{k \pi x}{p}-1, \\
& \text { (B.) } y_{x}^{(r)}=\frac{2^{x}}{p}+\frac{2}{p} \Sigma\left(2 \cos \frac{k \pi}{p}\right)^{x} \cos \frac{k \pi}{p}(x-2 r) ;
\end{aligned}
$$

la somme $\Sigma$ étant prise inclusivement depuis $k=1$ jusqu'à $k=\frac{1}{2} p-1$, si $p$ est un nombre pair, et jusqu'à $\frac{x}{2}(p-1)$ si $p$ est impair.

Démonstration. Soit $f(y)$ la fonction génératrice de $a_{r}$ en sorte que

$$
\text { 1. } f(y)=a_{0}+a_{1} y+a_{2} y^{2}+\ldots . .+a_{r} y^{r}+\ldots .
$$

Par la nature des racines $a, \alpha^{2}, \alpha^{3}, \ldots \ldots \alpha^{p}$ de l'équation $m^{p}-1=0$ on a 2. $a^{p-r} f(a y)+a^{2(p-r)} f\left(a^{2} y\right) \ldots+a^{p(p-r)} f\left(a^{p} y\right)=p\left(a_{r} y^{r}+a_{p+r} y^{p+r}+a_{2 p+r} y^{2 p+r}+\ldots\right)$. Donc si l'on observe que $y_{x}^{(r)}$ n'est que la somme des coëfficients de $y^{r}$, $y^{p+r}, y^{2 p+r}$ etc. dans le développement de la fonction $(1+y)^{x}-1$, on trouve en faisant d'abord $f(y)=(1+y)^{x}-1$, puis $y=1$ :

3. $y_{x}^{(r)}=\frac{1}{p}\left(\alpha^{p-r}(1+\alpha)^{x}+a^{2(p-r)}\left(1+\alpha^{2}\right)^{x}+\ldots .+\alpha^{p(p-r)}\left(1+\alpha^{p}\right)^{x}-\sum_{k=1}^{k=p} \alpha^{k(p-r)}\right)$. En faisant $a=\cos \frac{2 \pi}{p} \pm r(-1) \sin \frac{2 \pi}{p}$ on a

$$
\begin{aligned}
a^{k(p-r)} & =\cos \frac{2 k r \pi}{p} \mp r(-1) \sin \frac{2 k r \pi}{p}, \\
\left(1+\alpha^{k}\right)^{x} & =\left(2 \cos \frac{k \pi}{p}\right)^{x}\left(\cos \frac{k \pi x}{p} \pm \sqrt{ }(-1) \sin \frac{k \pi x}{p}\right), \\
a^{k(p-r)}\left(1+a^{k}\right)^{x} & =\left(2 \cos \frac{k \pi}{p}\right)^{x}\left(\cos \frac{k \pi}{p}(x-2 r) \pm r(-1) \sin \frac{k \pi}{p}(x-2 r)\right),
\end{aligned}
$$

dont il faut prendre la double-somme depuis $k=1$ jusqu'à $k=\frac{1}{2} p-1$ ou $\frac{I}{2}(p-1)$ (selon que $p$ est pair ou impair), ce qui fera nécessairement disparaître les imaginaires, puisque le résultat doit en être délivré. A cette somme si l'on ajoute le terme $2^{x}$, donné par $k=0$, on a la valeur de la série

$$
\alpha^{p-r}(1+\alpha)^{x}+\alpha^{2(p-r)}\left(1+\alpha^{2}\right)^{x} \ldots+\alpha^{p(p-r)}\left(1+\alpha^{p}\right)^{x},
$$

qu'il faut substituer dans l'équation (3.) pour avoir les deux équations $\left(A_{\text {. }}\right)$ 
et $(B$.$) , dont la différence est due au terme \sum_{k=1}^{k=p} \alpha^{k(p-r)}$, qui s'évanouit pour toutes les valeurs de $r$, excepté $r=0$ qui le rend égal à $p$.

Le principe, dont on fait ici usage, est le même qui a été employé dans plusieurs autres circonstances, par exemple par Mr. Poi isson dans sa belle théorie des séries périodiques (Journal de l'école polyt. $\left.19^{\text {ne }} c a h . p .421.\right)$, par Mr. A bel dans son premier mémoire sur la résolubilité des équations algébriques (tom. ${ }^{\text {er }}$ p. 72. de ce journal), par Mr. Jürg ensen dans ses "remarques sur une certaine transformation des fonctions" (tom. $6^{\text {ine }}$ p. 197. de ce journal).

Copenhague 20. septembre 1833. 